\title{
3D Structural Measurements of the Proximal Femur from 2D DXA Images Using a Statistical Atlas
}

\author{
Omar M. Ahmad ${ }^{\mathrm{a}}$, Krishna Ramamurthi ${ }^{\mathrm{b}}$, Kevin E. Wilson ${ }^{\mathrm{b}}, \mathrm{Klaus} \mathrm{Engelke}^{\mathrm{c}}$, Mary Bouxsein ${ }^{\mathrm{d}}$, \\ Russell H. Taylor ${ }^{\mathrm{a}}$ \\ ${ }^{\mathrm{a}}$ Johns Hopkins University, CISST, Baltimore MD; ${ }^{b}$ Hologic, Inc., Bedford MA; ${ }^{\circ}$ University of \\ Erlangen, Institute of Medical Physics, Erlangen, Germany; ${ }^{\mathrm{O}}$ Orthopaedic Biomechanics Laboratory, \\ BIDMC, Boston, MA.
}

\begin{abstract}
A method to obtain 3D structural measurements of the proximal femur from 2D DXA images and a statistical atlas is presented. A statistical atlas of a proximal femur was created consisting of both 3D shape and volumetric density information and then deformably registered to 2D fan-beam DXA images. After the registration process, a series of 3D structural measurements were taken on QCT-estimates generated by transforming the registered statistical atlas into a voxel volume. These measurements were compared to the equivalent measurements taken on the actual QCT (ground truth) associated with the DXA images for each of 20 human cadaveric femora. The methodology and results are presented to address the potential clinical feasibility of obtaining 3D structural measurements from limited angle DXA scans and a statistical atlas of the proximal femur in-vivo.
\end{abstract}

Keywords: Statistical Atlas, DXA, QCT, BMD

\section{INTRODUCTION}

\subsection{Osteoporosis}

Osteoporosis is defined as a skeletal disorder characterized by compromised bone strength predisposing one to an increased risk of fracture [1]. Bone strength is dependent upon both the density and 3-dimensional structure of the bone. Although fractures from osteoporosis can occur at various skeletal sites, fractures of the proximal femur often have the most serious consequences and are associated with the highest medical costs. $20 \%$ of senior citizens who have a hip fracture will die within a year from injury related complications. The annual direct medical care cost for osteoporotic fractures is approximately $\$ 15$ Billion dollars in the United States alone and hip fractures account for more than $1 / 2$ of that cost [2]. Thus early diagnosis of those at risk of hip fracture due to compromised bone strength is an area of intense research and the motivating subject-matter of this paper.

\subsection{Current Technology to Asses Skeletal Fragility}

Dual energy X-Ray absorptiometry (DXA) is considered the gold standard for the diagnosis of osteoporosis and the subsequent assessment of fracture risk. In DXA, two different X-ray spectra are used to obtain an image of a patient's mineral density of the bone (BMD g/ $\mathrm{cm}^{2}$ ). The average BMD of a region, e.g. the femoral neck, has been shown to predict decreased bone strength and fracture risk and thus is used clinically for the diagnosis of osteoporosis. However, because BMD contains no spatial information, and also because DXA is a projected measurement, it lacks important geometrical structural information of the mineral distribution, which may be of use in providing a more accurate strength estimate of the bone.

Another imaging technology, Quantitative Computed Tomography (QCT) is capable of producing 3D volumetric representations of the mineral density of bone. A vigorously researched hypothesis is that QCT can offer a better prediction for fracture risk over DXA. Although, due to the lack of prospective studies, the correctness of this hypothesis is at present not fully proven, QCT does allow for the direct measurement of 3D structural strength 
parameters (on a slice through the volume for example) such as, but not limited to, cross sectional area of the slice containing bone, and cross sectional moment of inertia. These measurements, however, are acquired at a cost; namely, the higher doses of radiation necessary to obtain a QCT scan and the general infrastructure and cost needed to support QCT-based assessment in a clinical setting.

\subsection{Using a Statistical Atlas to Obtain 3D Structural Information}

Our main contribution is the development of an alternative to QCT to obtain 3D structural information of a proximal femur by utilizing a statistical atlas and a limited number of DXA images. A relevant background of statistical atlases and $2 \mathrm{D}$ to $3 \mathrm{D}$ deformable registration is first presented which covers the methodology. We then present the 3D structural measurements which were taken from QCT-estimates generated from our statistical atlas and compare them to actual QCT measurements. Finally, we discuss the results and potential for creating a viable, lower-cost, lower-radiation, clinical alternative to QCT for obtaining structural parameters to assess skeletal fragility of the proximal femur.

\section{STATISTICAL ATLASES}

Statistical atlases of anatomical structures involve using prior information to create a mathematical model of a particular anatomy [3-7]. Presently, a male and a female statistical atlas were created using in-vivo segmented [8] QCT scans from 62 Caucasian men and 57 Caucasian women (The female femurs were highly osteoporotic). To begin building the female atlas, first a master QCT $Q_{\text {master }}$ was selected to be a reasonably average femur from the set of female QCT scans $Q_{1 \ldots} Q_{57}$. The binary segmentation mask for $Q_{\text {master }}$ was meshed [9] into a tetrahedral master mesh consisting of points $X_{\text {master }}$ and an index list $T$ defining the tetrahedron. $Q_{\text {master }}$ was 3D-3D deformably registered to each of the instances $Q_{1} \ldots Q_{57}$ to obtain a set of deformation fields $D_{1} \ldots D_{57}$ mapping voxels in $Q_{\text {master }}$ to $Q_{i}$. The deformation fields were applied to $X_{\text {master }}$ to obtain a set of deformed mesh instances points $X_{1} \ldots X_{57}$ associated with $Q_{1} \ldots Q_{57}$ ( All of the mesh instances have corresponding points and the same index list of tetrahedrons).

Each tetrahedron in each deformed mesh was overlaid onto its associated QCT scan $Q_{i}$ and the voxel values which lie in each tetrahedron were fit to a Bernstein polynomial model in the barycentric coordinate space of the tetrahedron. The polynomial fitting resulted in the generation of a list of coefficients, $\sigma_{i}=\left\{\beta_{i, 1} \ldots \beta_{i, j}\right\}$, associated with each subject mesh $\left(X_{i}\right.$ and $T$ ) parameterizing the polynomial density function for each tetrahedron. This allows for the generation of highly accurate simulated X-ray (DRR dynamically reconstructed radiograph) projections [10] and for the generation of a QCTlike voxel volume $V$ defined by the operator:

$$
\Omega(X, \sigma, T) \rightarrow V(x, y, z)
$$

To construct the female atlas, Initially all of the point sets were laid out as $3 k$ element vectors where $k$ is the number of points:

$$
X=\left(x_{1}, \ldots, x_{k}, y_{1}, \ldots, y_{k}, z_{1}, \ldots, z_{k}\right)^{T} \text {. }
$$

Following the general approach of Cootes [3], an iterative process was performed in which each instance was translated so that its center of gravity was at the origin. The first instance $X_{I}$ was chosen as an initial estimate of the mean shape $\bar{X}$. All of the shapes $X_{2 \ldots 57}$ were aligned to this current mean shape through a similarity transform. The mean shape $\bar{X}$ was re-estimated from the aligned shapes: 


$$
\bar{X}=\frac{1}{57} \sum_{i=1}^{57} X_{i}
$$

This process was repeated until convergence. A data matrix $D$ was then constructed,

$$
D=\left|\begin{array}{c}
X_{1}-\bar{X} \\
\vdots \\
X_{57}-\bar{X}
\end{array}\right|
$$

and Principal Component Analysis performed by taking the singular value decomposition of $D$,

$$
D=U \Lambda V^{T}
$$

The first 57 vectors of $V$ are denoted as $\Phi_{1 \ldots 57}$, and comprise the orthogonal modes of variation Figure 1 . The mean shape $\bar{X}$ and the modes $\Phi_{1 \ldots 57}$ together form the statistical atlas. It is hypothesized that for some shape represented by the point set $X_{c}$, sharing the same point correspondences as $\bar{X}$ and $X_{1 \ldots 57}$, we can approximate $X_{c}$ by

$$
X_{c} \cong F_{s}\left(\bar{X}+\sum_{i=1}^{57} \lambda_{i} \Phi_{i}\right)
$$

where $F_{s}$ is a similarity transformation and $\lambda_{i}$ are scalar mode weights. However, the QCT volumes will be approximated directly and therefore $e q .6$ is modified with $e q .1$ such that for some subject QCT volume $Q_{\text {subject }}$ we can approximate it by $Q_{\text {est }}$.

$$
Q_{\text {subject }} \cong\left[Q_{\text {est }}=\Omega\left(F_{s}\left(\bar{X}+\sum_{i=1}^{57} \lambda_{i} \Phi_{i}\right), \quad \bar{\sigma}, T\right)\right] .
$$

The process for creating the male atlas followed the female with two important distinctions. First, 62 very healthy subjects were used. Secondly, the corresponding master shape was converted into a spring-mass system. One side of the vertices corresponding to edge intersections of tetrahedron crossing the boundary of trebecular bone to cortex were marked and vertices shifted to "lock" onto the inner cortex and then regularized. This was done to isolate a smoother cortical exterior in the shape model. This was not performed on the female model for the osteoporotic irregularities precluded a smooth regularization. 


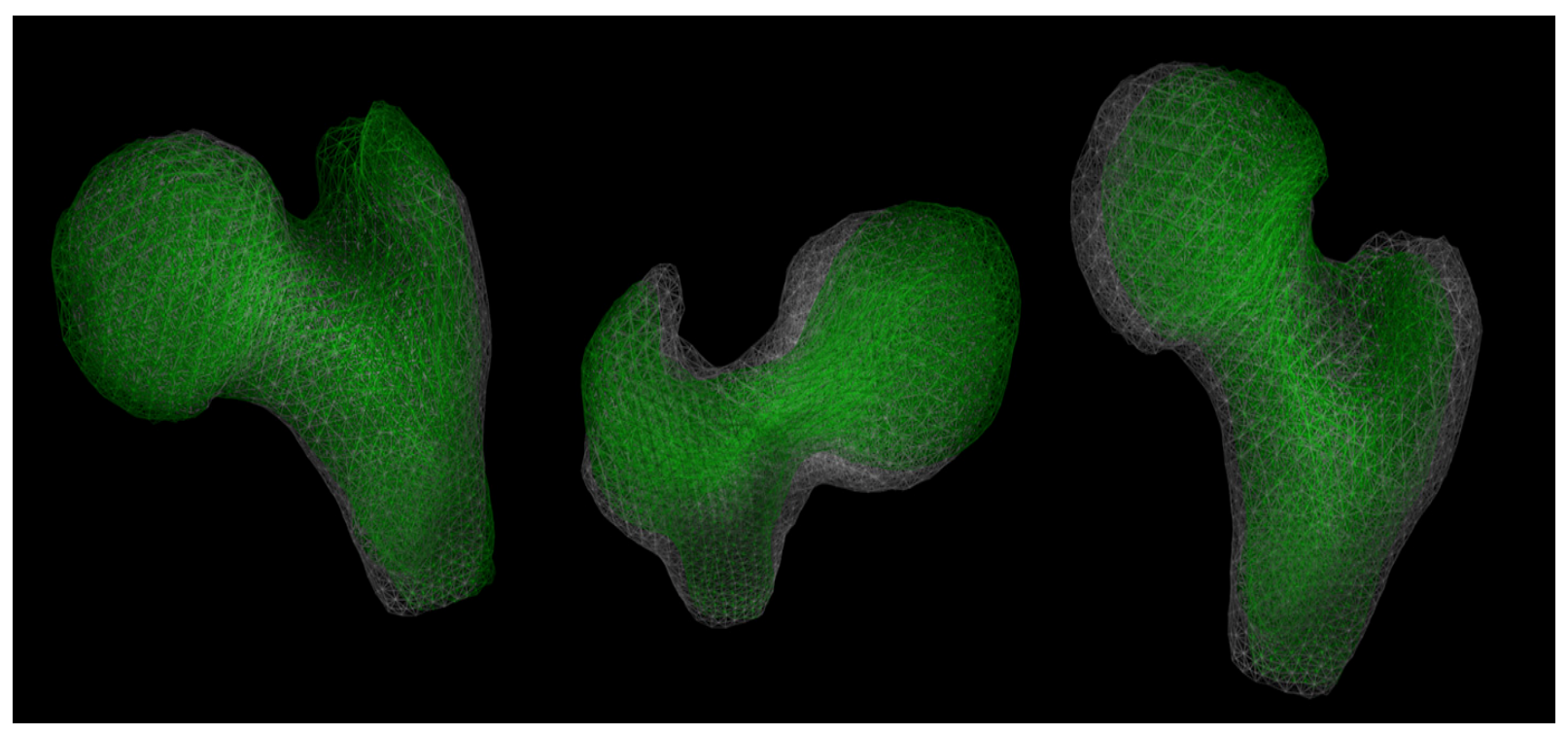

Fig. 1. The mean shape is shown in gray. Superimposed in green are the modes ( $\lambda=120$ for illustration) added to the mean shape: $\bar{X}+\lambda \Phi_{3}$ (left), $\bar{X}+\lambda \Phi_{2}$ (middle), and $\bar{X}+\lambda \Phi_{1}$ (right).

\section{2D-3D REGISTRATION}

PCA is a dimensionality reduction technique and thus it is assumed that a small subset $r$ of the modes $\left(\Phi_{l \ldots r}\right)$ account for a relative majority of the individual shapes' anthropomorphic variations. In theory, it should be possible to produce a QCT-estimate volume $Q_{e s t}$ of a subject QCT volume $Q_{\text {subject }}$ from the modes $\Phi_{1 \ldots r}$ with limited additional data of the subject. In the present experiment, the limited additional data comprised 4 real DXA scans of the subject. The projection angles were chosen to minimize pelvic overlap and obtainable in-vivo. The problem can now be stated specifically: for a given cadaveric subject with 4 DXA images, and an associated measured QCT data set $Q_{\text {subject }}$, what are the values of $F_{s}$ and $\lambda_{1 \ldots r}$, derived from the DXA images alone, which minimize the error between $Q_{\text {subject }}$ and $Q_{\text {est }}$.

In order to ascertain $F_{s}$ and $\lambda_{1 \ldots r}$ to build an estimate $Q_{e s t}$, an iterative minimization process [4, 11] was followed whereby the 4 DXA projections were compared to DRR's of the gender-specific statistical atlas with a mutual information metric $[11,12]$. Yao [4] was augmented by applying a simulated annealing process to better avoid local minima. The rotation, scale, translation, $\left(F_{s}\right)$ and the scalar weights $\lambda_{1 \ldots 12}$ for the first 12 modes (12 were chosen by looking at the eigenvalues (eq. 5) to account for $>90 \%$ of the variation) of variation were varied on the statistical atlas to minimize the error between the simulated DRR projections of the model and the actual DXA images. The process is graphically depicted in Figure 2 and was done for each the 20 cadaver subjects (For the 2D-3D registration process, the gender specific atlas was used for each cadaver). The final registration parameters $\left(F_{s}\right.$ and $\left.\lambda_{1 \ldots 12}\right)$ were stored for each cadaver. An estimate $Q_{\text {est }}$ was then created for each cadaver. 
(e) Vary rotation.
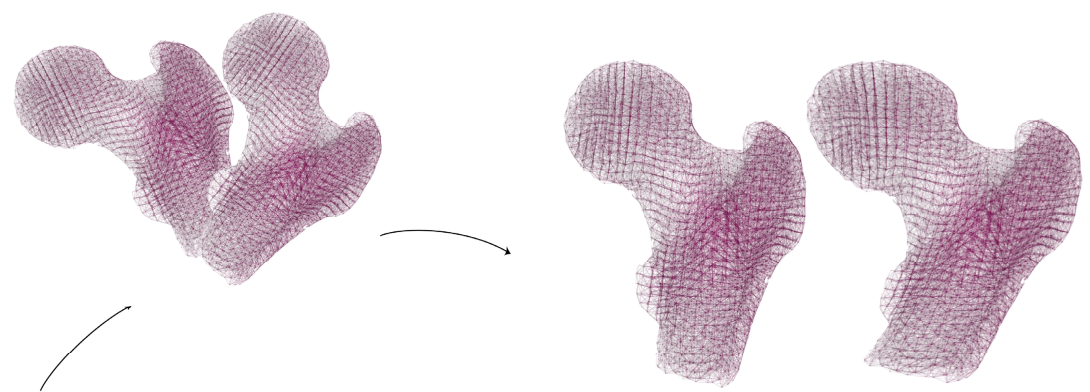

(f) Vary shape modes.

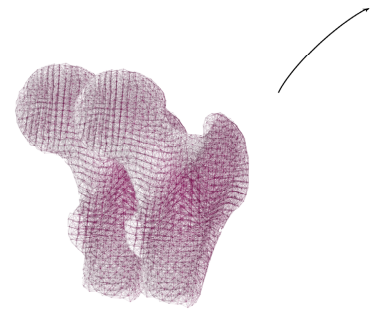

(d) Vary translation.

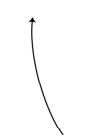

$$
\text { . }
$$




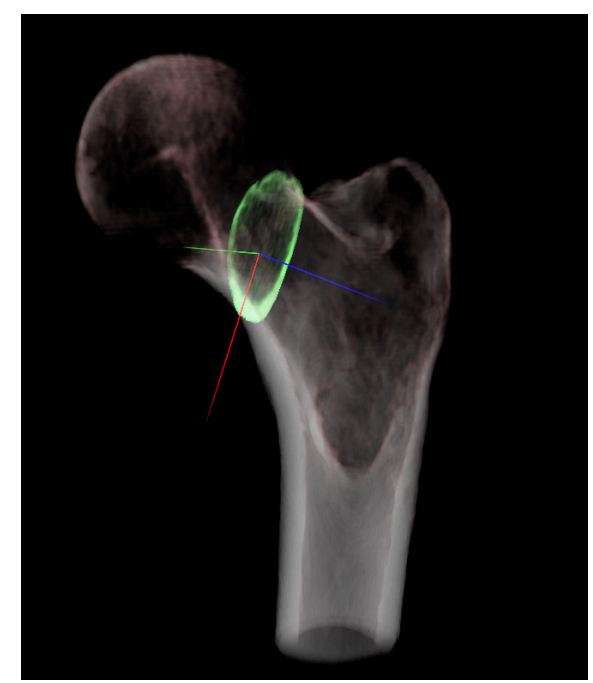

Fig. 3. Shown is a slice of interest in the mid-neck region upon which a series of structural measurements were taken on the QCT and QCT estimates.

\section{FAN-BEAM CORRECTION}

\subsection{GPU DRR Projections}

An important contribution in the scope of this work was taking the method of Sadowski [10] and altering it to create fan beam DRRs as opposed to cone beam. The algorithm involves rendering a tetrahedron in triangular parts, where in each triangular part, the front and back intersections of a line passing through the tetrahedron to the eye-point can be interpolated in the GPU for every pixel comprising the triangle. Given these barycentric entrance and exit points (per pixel), a closed form solution can be calculated from the Bernstein polynomial coefficients, $\sigma_{i}=\left\{\beta_{i, 1} \ldots \beta_{i, j}\right\}$, for the attenuation value of the line integral through the tetrahedron.

\subsection{Fan-Beam Modification}

In a fan beam projection, a point is projected perspectively along one axis and orthographically along another. Moreover, there is no source "point", but rather, a source "line". The correct point projections are achieved by explicitly changing the OpenGL "model-view-projection" matrix and only dividing by $\mathrm{z}$ along the horizontal $\mathrm{x}$ axis. This generates the correct fan-beam point geometry. To define a single source point, the projection source point is moved for each tetrahedron along its vertical axis to be at the average height of the vertices of the tetrahedron. This introduces a very small non-linearity in the rasterization process since a line in 3 dimensional space projects with a very slight curve. However, the overall effect is negligible and the change produces an accurate "fan-beam" projection. 


\section{RESULTS}

To test the fidelity of the present approach, 2D-3D registrations (Figure 2) were performed on DXA's of the proximal femurs taken from 20 cadaveric femora (11 women, 9 men, age 62 to 95). The resulting statistical models were then converted into QCT-estimate volumes. We examined the association between QCT-estimate and QCT derived femoral neck length (FNL), volumetric density of the total proximal femur (vBMD), as well as the area of bone mineral in the cross section $\left(\mathrm{CSA}_{\mathrm{b}}\right)$ and the mass-weighted polar moment of inertia $\left(\mathrm{CSMI}_{\mathrm{p}}\right)$, computed at a corresponding QCTestimate and QCT slice at the mid-neck (Figure 3). $\mathrm{CSA}_{\mathrm{b}}$ is the resistance to axial compression and $\mathrm{CSMI}_{\mathrm{p}}$ is related to resistance bending. vBMD detangles geometry and density (combined in DXA BMD) which is necessary to calculate structural properties. FNL is related to HAL (Hip Axis Length) which is currently being studied as a fracture predictor. We found very strong linear correlation between the QCT-estimate and QCT for all parameters (Table 1 and Figure 4):

Table 1. Measurements which were taken on both the QCT and the QCT-estimates with their associated units, descriptions, and $\mathrm{r}$ values.

\begin{tabular}{lllc}
\hline Measurement & Units & Description & r Value \\
\hline vBMD & $\mathrm{g} / \mathrm{cm}^{3}$ & Volumetric Bone Mineral Density & $\mathbf{9 3}$ \\
CSA $_{\mathrm{b}}$ & $\mathrm{cm}^{3}$ & Bone Percentage Weighted Cross Sectional Area. & $\mathbf{9 6}$ \\
CSMI $_{\mathrm{p}}$ & $\mathrm{g} \mathrm{cm}$ & Polar Cross Sectional Moment of Inertia & $\mathbf{9 4}$ \\
FNL & $\mathrm{mm}$ & Femoral Neck Length & $\mathbf{9 7}$
\end{tabular}

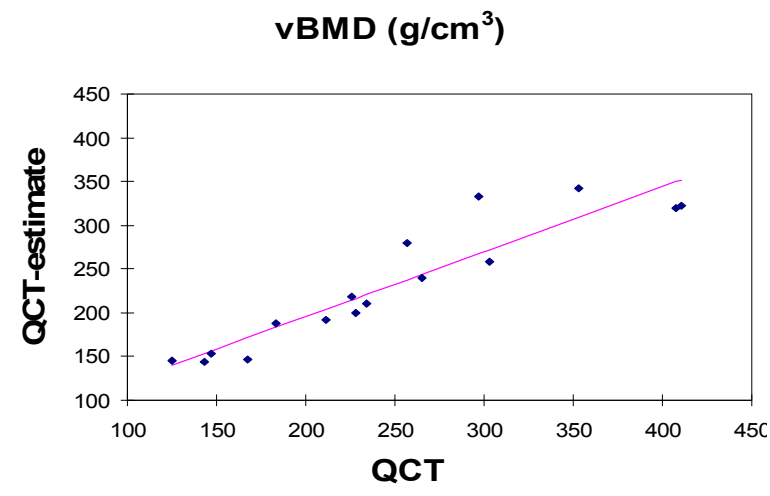

$\operatorname{CSA}\left(\mathrm{cm}^{2}\right)$

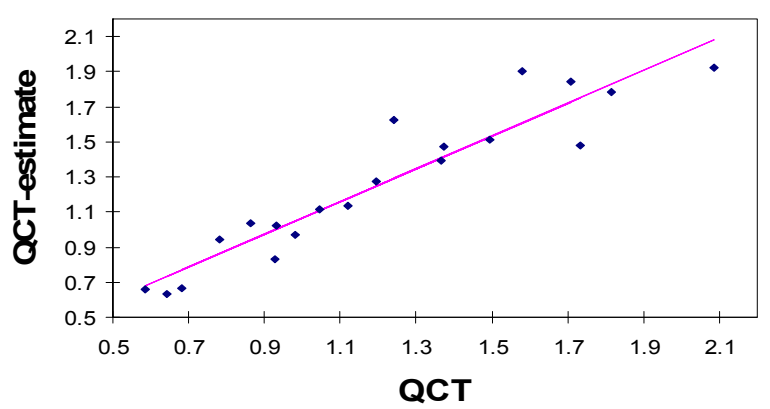

Femoral Neck Length (mm)

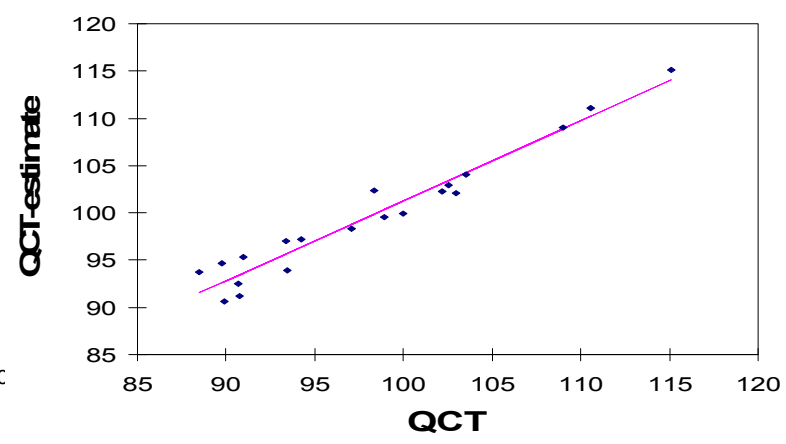

$\operatorname{CSMI}\left(\mathrm{g} \mathrm{cm}^{2}\right)$

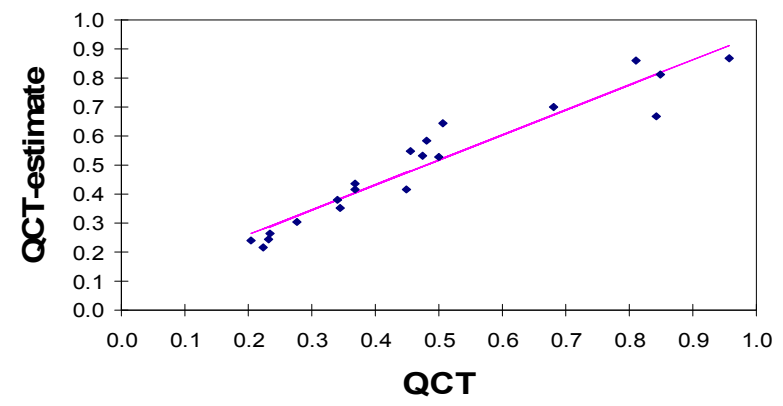

Fig. 4. The corresponding plots to table 1. 


\section{CONCLUSIONS AND FUTURE WORK}

The correlation $r$ values obtained by measuring FNL, vBMD, $\mathrm{CSA}_{b}$, and $\mathrm{CSMI}_{\mathrm{p}}\left(\mathrm{CSA}_{\mathrm{b}}\right.$, and $\mathrm{CSMI}_{\mathrm{p}}$ are measured on a mid-neck slice of interest), between QCT and the QCT-estimates are reasonably high to lead us to conclude that there is in fact clinical feasibility in obtaining 3D structural measurements from 2D DXA and a statistical atlas of the proximal femur. However, some limitations of this work may be mentioned, including a relatively small number of the samples used for validation, which probably does not reflect the total variability in the femoral geometry and especially agingrelated factors. Therefore, further experiments and research is needed to demonstrate the same results on in-vivo data with a larger sample set. Promising experiments are currently being conducted as such.

\section{REFERENCES}

1. Definition from the NIH 2000 consensus statement. http://consensus.nih.gov/2000/2000Osteoporosis111html.htm (2000)

2. Bone Health and Osteoporosis, a Report of the Surgeon General. Department of Health and Human Services. (2004)

3. Cootes, T., Taylor, C., Cooper, D., Graham, J.: Active shape models - their training and application. Computer Vision Image Understanding 61(1), 38-59 (1995)

4. Yao, J. A statistical bone density atlas and deformable medical image registration. Ph.D. thesis, Johns Hopkins University (2002)

5. Wu, C., Murtha, P.E., Mor, A.B., Jaramaz, B.: A two-level method for building a statistical shape atlas. In: CAOS (2005)

6. Sadowsky, O., Ramamurthi, K., Ellingsen, L., Chintalapani, G., Prince, J., Taylor, R.: Atlas assisted tomography: registration of a deformable atlas to compensate for limited-angle cone-beam trajectory. In: IEEE International Symposium for Biomedical Imaging pp. 1244-1247 (2006)

7. Cootes, T., Beeston, C., Edwards, G., Taylor, C.: A unified framework for atlas matching using active appearance models. In: IPMI, pp. 322-333 (1999)

8. Y. Kang, K. Engelke, W. Kalender.: A New accurate and precise 3D segmentation method for bone in volumetric CT data. In: IEEE Trans. Med. Ima., 22:586:598, (2003)

9. Mohamed, A., Davatzikos, C.: An approach to 3D finite element mesh generation from segmented medical Images. In: IEEE ISBI (2004)

10. Sadowsky, O., Cohen, J., Taylor, "Rendering Tetrahedral Meshes with Higher-order Attenuation Functions for Digital Radiograph Reconstruction," in Proceedings of IEEE Visualization 2005.

11. L Zollei, E Grimson, A Norbash, and W Wells. 2D-3D registration of x-ray fluoroscopy and ct images.: in IEEE CVPR, (2001)

12. Viola, P., and Wells, W.M.: Alignment by maximization of mutual information. In: International Journal of Computer Vision, vol. 24, pp. 137-154, (1997) 